\title{
Binary opinion dynamics with noise on random networks
}

\author{
CHI LiPing \\ Institute of Particle Physics \& Complexity Science Research Center, Central China Normal University, Wuhan 430079, China
}

Received April 18, 2011; accepted May 31, 2011

\begin{abstract}
Two kinds of noise strategies in binary opinion dynamics on ER random networks are discussed. Random noise $p_{1}$ in the initial configuration plays a role in redistributing the opinion states associated with the network. Under synchronous updating, the system can attain a stable state within few time steps. The fraction of nodes with changed opinion states $F$ decreases exponentially with time, and the ratio of one of the two opinion states $R$ remains almost unchanged during the evolution. The average ratio $<R>$ crosses at the half-half initial concentration under different $p_{1}$. For noise in the dynamical evolution $p_{2}$, the system can reach a steady state with small fluctuations. With larger $p_{2}$, more nodes have changed opinion states at each updating and more nodes with opposite opinions coexist. If $p_{2}$ is greater than 0.5 , the two opinions coexist with equal support.
\end{abstract}

binary opinion dynamics, random noise, complex networks

Citation: Chi L P. Binary opinion dynamics with noise on random networks. Chinese Sci Bull, 2011, 56: 3630-3632, doi: 10.1007/s11434-011-4751-1

In recent years, much effort has been invested in social dynamics formulated with concepts and methods from statistical physics [1-3]. Opinion dynamics is one of the social problems well-studied by physicists based on the famous Ising model from three decades ago [4]. Since then, quite a few opinion models have been proposed, such as the voter model [5,6], the Sznajd model [7-13] and the bounded confidence model [14-16], to name a few. In most of these models, each of $N$ agents is assigned a finite number of available states of opinions. Opinion formation is modeled as a collective behavior of agents in which individuals evolve following either majority rule or imitation. In a binary opinion dynamics, two competing states, +1 or -1 , are considered. Despite the complex dynamics of opinions among agents, the system attains total consensus of one of the two contrasting opinions, or a steady state with an equal distribution of opinions.

The growing field of complex networks [17-22] enables us to obtain a better knowledge of social systems. The intense theoretical research currently taking place, examines the systems of nodes representing agents and links repre-

email: chilp@mail.ccnu.edu.cn senting the interactions between them. In the random network proposed by Erdos and Renyi (ER), $N$ nodes are connected by $n$ edges selected randomly from the $N(N-1) / 2$ possible edges; the connection probability is $p=n / N(N-1) / 2$. The number $k$ of edges connecting one node to others is called the degree of that node. The average degree of the random network is $<k>=2 n / N=p(N-1) \approx p N$ if $p<<1$.

The aim of our model in this paper is to explore the evolution and to determine the final state of the binary opinion dynamics with noise. The opinion dynamics is performed on the ER random network with nodes $N=10^{5}$ and average degree $<k>=4$. In the initial configuration, each node is assigned an opinion -1 with probability $f$ and +1 with probability $1-f$. In this binary opinion dynamics, individuals evolve by majority rule: the sign of each node is determined according to the majority of its nearest neighbors. Two kinds of noise strategies are discussed: (i) noise in the initial configuration; and (ii) noise in the dynamical evolution.

We stress that the evolution in the paper is synchronous, which means that at each iteration, every node in the network calculates its next state depending on neighborhood opinions, thus to ensure all nodes take the majority opinions in their groups. 
First, we consider the noise in the initial configuration. We randomly choose nodes with probability $p_{1}$ to take their opposite states before the evolution. With synchronous iteration under majority rule, we find that the system attains a stable state within a few steps [8]. In the stable state, all nodes are in the majority compared with their nearest neighbors and maintain their opinions. We use $F$ to denote the fraction of nodes that have changed their opinion states at any given iteration, i.e. $F=N_{\text {changed }} / N$.

The smaller $F$ is the more stable the system becomes. Figure 1 shows $F$ evolving with the simulation time under different probabilities $p_{1}$. The fraction of nodes with changed states has no relevant to $p_{1}$, but depends exponentially on time $t$, with the exponent about -1.75 .

To obtain the time-dependence of the opinion states, we record the ratio of opinions -1 within the system after each update here denoted by $R=N_{-1} / N$.

The inset in Figure 2 plots the time variation of $R$ when the initial fraction of opinion -1 is 0.1 , that is, $f=0.1$. We find that $R$ remains almost unchanged under different $p_{1}$. We also notice that the ratio of opinion -1 can rise to as high as 0.5 in the final state under $p_{1}=0.7$ even though the initial fraction is $f=0.1$. Assuming $R$ is unrelated to $p_{1}$, we record the average value $\langle R\rangle$ after 10 realizations with each $p_{1}$ with fixed $f$. Figure 2 shows the results of $\langle R>$ as a function of $f$. The intersection of different $p_{1}$ is made at $f=$ 0.5 . If $f<0.5<R>$ becomes larger with increasing $p_{1}$, whereas if $f>0.5<R>$ decreases with $p_{1}$.

These results can be explained by considering that if we change the states of randomly chosen nodes, the initial distribution of opinions is changed correspondingly. After introducing $p_{1}$, the fraction of opinion -1 in the initial distribution takes the form $f\left(1-p_{1}\right)+(1-f) p_{1}$, in which the first term is the probability of staying in state -1 and the second term is the probability due to transitions from state +1 to -1 .

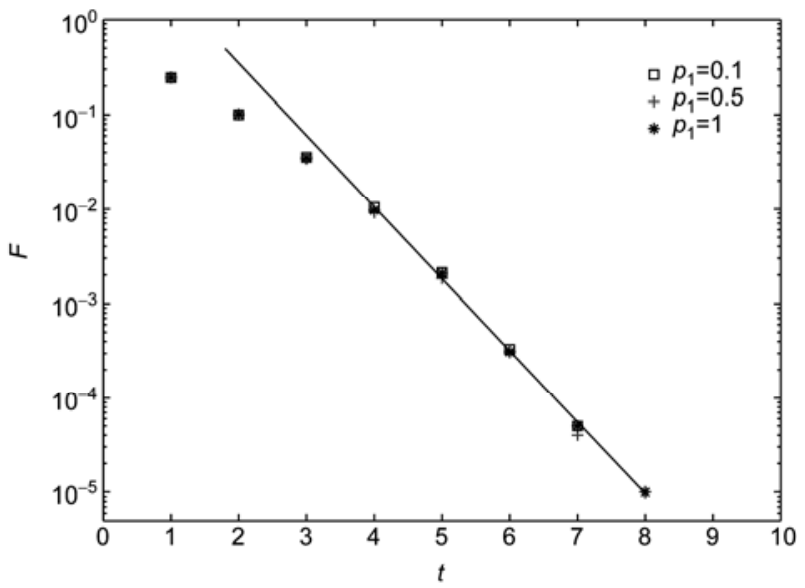

Figure 1 Time evolution of the fraction of nodes with changed opinion states. For the random noise in the initial configuration, the fraction $F$ decreases exponentially with $t$ under probability $p_{1}=0.1,0.5,1$, respectively. The exponent is about -1.75 .

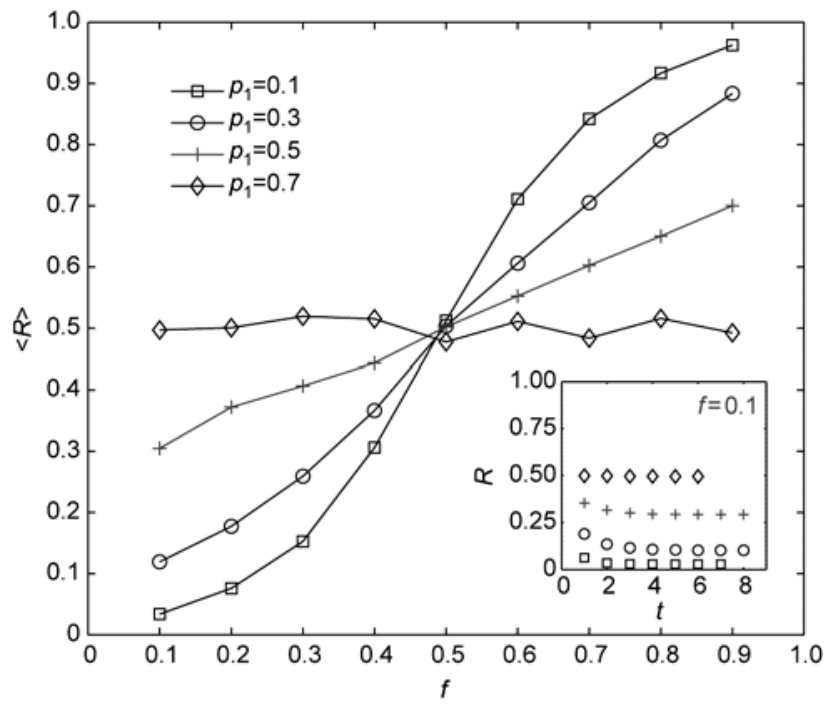

Figure 2 Plot of the average ratio of opinion $-1,\langle R>$, as a function of $f$ under different probabilities of $p_{1}$ for the noise in the initial configuration. The inset shows $R$ evolving with $t$ when $f=0.1$. The lines are for eye guidance.

It is obvious that the configuration of the network will not be changed at $f=0.5$ under different $p_{1}$. The application of random noise $p_{1}$ shifts the initial concentration of opinion -1 from $f$ to $f\left(1-p_{1}\right)+(1-f) p_{1}$. The effect of $p_{1}$ is corresponding to the redistribution of initial opinion states.

Next, we discuss the noise in the dynamical evolution. In this noise strategy, at each synchronous updating, we choose nodes with probability $p_{2}$ that stay at their minority states compared with their nearest neighbors and with probability $1-p_{2}$ that the nodes assume the majority states. For $p_{2}=0$, the model results in ordinary majority rule on random networks.

Figure 3 shows the time variation of $R$ and $F$ given $f=0.5$ with $p_{2}=0.01,0.1,0.5,0.7$. We find that the system can arrive at a steady state with small fluctuations. If $p_{2}$ is very small, see $p_{2}=0.01$ in Figure 3, the system has a tendency towards a state of consensus. As $p_{2}$ becomes larger, more nodes change their opinion state and hence more nodes with opposing opinions exist in the system. If $p_{2} \geqslant 0.5$, the two opposing opinions coexist with equal support. At the same time, more than half of the nodes keep changing their opinions.

Recall that noise parameter $p_{2}$ represents the fraction that nodes will not adopt majority states of their nearest neighbors, but keep their minority states. If we introduce noise into the dynamics of majority rule, the system can no longer reach a consensus state. With $p_{2} \rightarrow 0$, the system will be nearing the consensus state. If $p_{2} \geqslant 0.5$, most nodes intending to change will keep their minority states, whereas the rest evolve under majority rule. As a result, the system will retain thereabouts equal support for the two opinions.

In summary, we have investigated two kinds of noise strategies in binary-opinion dynamics for ER random networks. The random noise $p_{1}$ in the initial configuration has 

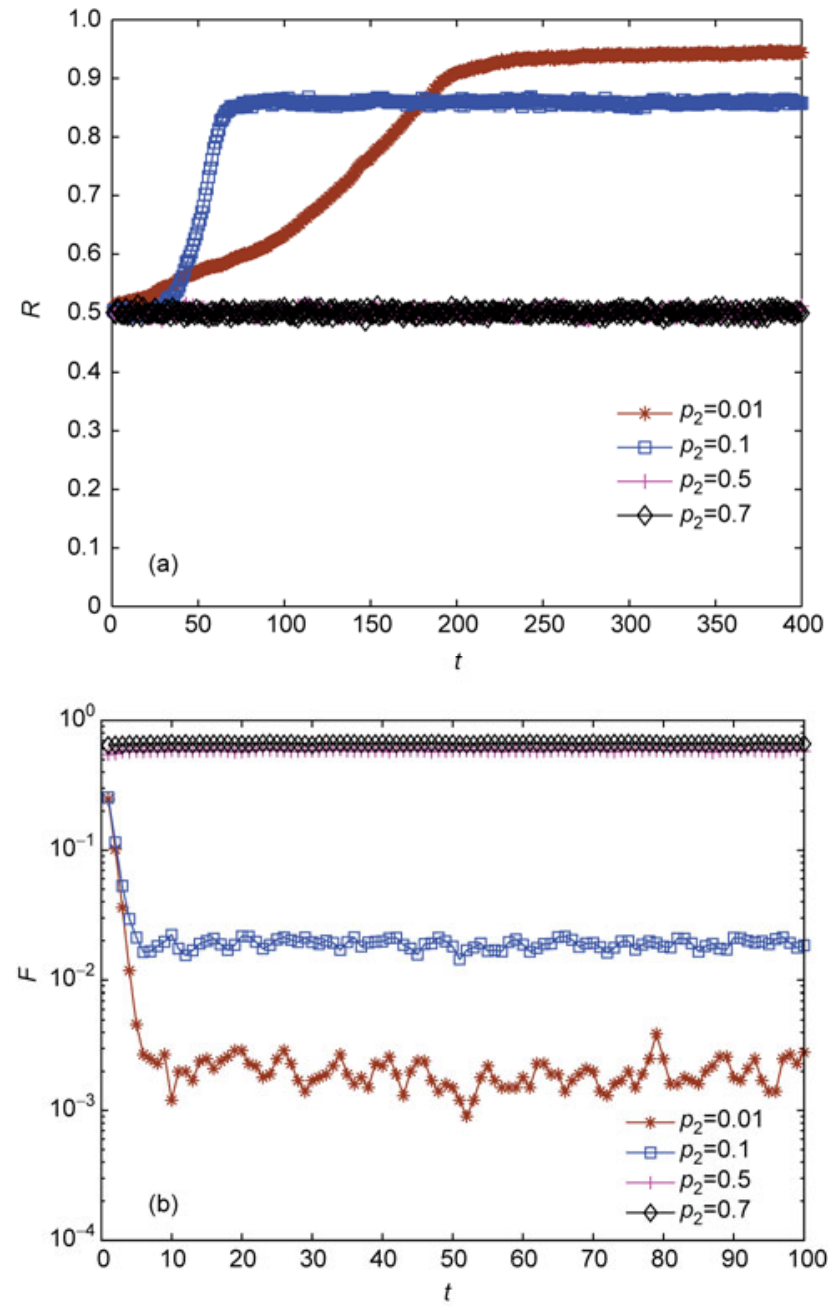

Figure 3 The ratio of opinion $-1 R$ (a) and the fraction of changed nodes $F$ (b) as a function of time step $t$ for the noise in the dynamical evolution.

played a role in the redistribution of opinion states in the network. Under synchronous updating, the system attained a stable state within a few time steps. The fraction of nodes with changed opinion states $F$ falls off exponentially with time. The ratio $R$ of one of the two opinion states stayed almost unchanged during the evolution. The average ratios $<R>$ for different $p_{1}$ all crossover at $f=0.5$. For noise $p_{2}$ in the dynamical evolution, the system can arrive at a steady state with small fluctuations. With larger $p_{2}, F$ had become larger and more nodes with opposing opinions coexisted in the system. If $p_{2}$ is greater than 0.5 , the two opinions coexist with equal support with more than half of the nodes changing state, i.e. opinions, within any time step.

This work was supported in part by the National Natural Science Foundation of China (10635020 and 10975057) and the Ministry of Education of China (306022).

1 Castellano C, Fortunato S, Loreto V. Rev Mod Phys, 2009, 81: 591-646

2 Li T, Zhang J F. Sci China Ser F-Inf Sci, 2009, 52: 2089-2103

3 Zhong S B, Huang Q Y, Song D J. Sci China Ser D-Earth Sci, 2009, 52: $550-561$

4 Galam S, Gefen Y, Shapir Y. J Math Sociol, 1982, 9: 1-13

5 Holly R A, Liggett T M. Ann Probab, 1975, 3: 633-643

6 Castello X, Toivonen R, Eguiluz V M, et al. Europhys Lett, 2007, 79: 66006

7 Sznajd-Weron K, Sznajd J. Int J Mod Phys C, 2000, 11: 1157-1165

8 Shao J, Havlin S, Stanley H E. Phys Rev Lett, 2009, 103: 018701

9 Stauffer D, Sousa A O, Oliveira S M. Int J Mod Phys C, 2000, 11: 1239-1245

10 Chang I. Int J Mod Phys C, 2001, 12: 1509-1512

11 He M, Li B, Luo L. Int J Mod Phys C, 2004, 15: 997-1003

12 Bartolozzi M, Leinweber D B, Thomas A W. Phys Rev E, 2005, 72: 046113

13 Rodrigues F A, Costa L F. Int J Mod Phys C, 2005, 16: 1785-1792

14 Deffuant G, Neau D, Amblard F, et al. Adv Comp Syst, 2000, 3: 87-98

15 Fortunato S. Int J Mod Phys C, 2004, 15: 1301-1307

16 Ben-Naim E, Krapivsky P L, Redner S. Physica D, 2003, 183: 190-204

17 Albert R, Barabasi A L. Rev Mod Phys, 2002, 74: 47-97

18 Newman M E J. SIAM Rev, 2003, 45: 167-256

19 Watts D J, Strogatz S H. Nature, 1998, 393: 440-442

20 Barabasi A L, Albert R. Science, 1999, 286: 509-512

21 Liu H T, Li W W. Chinese Sci Bull, 2010, 55: 3458-3465

22 Chen W J, Li X N, Feng X, et al. Chinese Sci Bull, 2010, 55: 2531-2538

Open Access This article is distributed under the terms of the Creative Commons Attribution License which permits any use, distribution, and reproduction in any medium, provided the original author(s) and source are credited. 